\section{OPEN ACCESS}

Edited by:

Corin Badiu,

Carol Davila University of Medicine and Pharmacy, Romania

Reviewed by:

Sally A. Camper,

University of Michigan, United States

Ann McCormack,

St Vincent's Hospital

Sydney, Australia

${ }^{*}$ Correspondence:

Bartlomiej Budny

bbudny@ump.edu.pl

Specialty section:

This article was submitted to Pituitary Endocrinology,

a section of the journal

Frontiers in Endocrinology

Received: 08 January 2020

Accepted: 11 May 2020

Published: 16 June 2020

Citation:

Budny B, Zemojtel T, Kaluzna M, Gut $P$, Niedziela $M$,

Obara-Moszynska M,

Rabska-Pietrzak B,

Karmelita-Katulska K, Stajgis M

Ambroziak U, Bednarczuk T, Wrotkowska E, Bukowska-Olech E,

Jamsheer $A$, Ruchala $M$ and

Ziemnicka K (2020) SEMA3A and IGSF10 Are Novel Contributors to

Combined Pituitary Hormone Deficiency (CPHD).

Front. Endocrinol. 11:368. doi: 10.3389/fendo.2020.00368

\title{
SEMA3A and IGSF10 Are Novel Contributors to Combined Pituitary Hormone Deficiency (CPHD)
}

\begin{abstract}
Bartlomiej Budny ${ }^{1 *}$, Tomasz Zemojtel ${ }^{2,3}$, Malgorzata Kaluzna ${ }^{1}$, Pawel Gut ${ }^{1}$, Marek Niedziela ${ }^{4}$, Monika Obara-Moszynska ${ }^{4}$, Barbara Rabska-Pietrzak ${ }^{4}$, Katarzyna Karmelita-Katulska ${ }^{5}$, Marek Stajgis ${ }^{5}$, Urszula Ambroziak ${ }^{6}$, Tomasz Bednarczuk ${ }^{6}$, Elzbieta Wrotkowska ${ }^{1}$, Ewelina Bukowska-Olech ${ }^{7}$, Aleksander Jamsheer ${ }^{7}$, Marek Ruchala ${ }^{1}$ and Katarzyna Ziemnicka ${ }^{1}$
\end{abstract}

1 Department of Endocrinology, Metabolism and Internal Diseases, Poznan University of Medical Sciences, Poznan, Poland, ${ }^{2}$ Institute for Medical and Human Genetics, Charité-Universitätsmedizin Berlin, Berlin, Germany, ${ }^{3}$ Genomics Platform, Berlin Institute of Health, Berlin, Germany, ${ }^{4}$ Department of Pediatric Endocrinology and Rheumatology, Poznan University of Medical Sciences, Poznan, Poland, ${ }^{5}$ Department of General Radiology and Neuroradiology, Poznan University of Medical Sciences, Poznan, Poland, ${ }^{6}$ Department of Internal Medicine and Endocrinology, Medical University of Warsaw, Warsaw, Poland, ${ }^{7}$ Department of Medical Genetics, Poznan University of Medical Sciences, Poznan, Poland

Background: The mutation frequencies of pituitary transcription factors genes in patients with combined pituitary hormone deficiencies (CPHD) vary substantially between populations. However, apart from PROP1 the mutation rate of other genes is low and for almost half of the patients with CPHD the routine sequencing of known genes is unsuccessful in the identification of genetic causes.

Methods: A cohort of 66 sporadic and nine familial CPHD cases (80 patients in total) were subjected to initial testing of the genes PROP1, POU1F1, LHX3, LHX4, and HESX1 using a targeted gene panel and MLPA. In patients who tested negative, a whole exome sequencing approach was employed.

Results: In nine of the familial cases and 32 of the sporadic patients mutations in the PROP1 gene were found (the common pathogenic variants included c.301_302delAG and c.150delA). Mutations were also found in genes so far not related directly to CPHD. A unique homozygous and clinically relevant variant was identified in the SEMA3A gene, which may contribute to neural development and his phenotypic spectrum including short stature and isolated hypogonadotropic hypogonadism $(\mathrm{IHH})$. Another pathogenic variant p.A1672T was found in the IGSF10 gene reported to be responsible for delayed puberty and neuronal migration during embryogenesis. Several suspected novel but predicted benign variants were also identified for the CHD7, WDR11 and FGF17 genes.

Conclusion: Although PROP1 defects account for a majority of CPHD patients, identification of rare, less frequent variants constitutes a big challenge. Multiple genetic factors responsible for CPHD are still awaiting discovery and therefore the usage of efficient genomic tools (i.e., whole exome sequencing) will further broaden our knowledge regarding pituitary development and function.

Keywords: pituitary, CPHD, PROP1, SEMA3A, IGSF10, CHD7 


\section{INTRODUCTION}

Combined Pituitary Hormone Deficiency (CPHD) is caused by both genetic and nongenetic factors including trauma, tumor, infection, irradiation, and autoimmune diseases. The prevalence of CPHD was recently estimated to be 1 in 8,000 individuals worldwide (Genetics Home Reference at NIH, https://www.ghr.nlm.nih.gov). The genetic defects causing CPHD typically result in misdevelopment of the anterior pituitary gland and insufficient hormone secretion, which manifests in early childhood. So far $\sim 30$ genes have been found to cause CPHD in its nonsyndromic or more severe syndromic forms (1). The most frequently mutated genes belong to the transcription factors family including PROP1, HESX1, LHX3, LHX4, POU1F1, GLI2, OTX2 but mutations within those genes would explain only some cases (2). The entire picture of pituitary hormone deficiency is furthermore emphasized by its associated severe syndromic phenotypes including craniofacial defects i.e., septo-optic dysplasia SOD (HESX1, OTX2, GLI2, GLI3) or holoprosencephaly $(S H H)$. Recently, the phenotypic spectrum of $\mathrm{CPHD}$ has broadened due to the reported overlapping manifestation with isolated hypogonadotropic hypogonadism (IHH). This was somewhat expected since both conditions may share similar etiologies. The co-occurrence of IHH in CPHD phenotype was already well proven in regards to the GLI2, $S O X 2$, and SOX 3 genes and recently pituitary dysfunction was confirmed for well characterized IHH genes namely CHD7, PROKR2, FGFR1, and FGF8 (3). The recent improvement of genetic testing technologies and the possibility of screening all genes within a patient should quickly be beneficial in broadening the list of CPHD-IHH overlapping phenotypes, particularly for the gonadotropin related genes but also others contributing to the hypothalamic-pituitary axis.

This study reports a comprehensive genetic examination of participating CPHD patients to identify defects in previously known genes as well as genes at new loci in order to recognize novel genetic mechanisms of the development of the CPHD.

\section{PATIENTS}

The present study included a total of 80 patients with CPHD. All participating patients were diagnosed according to standard procedures in the Department of Pediatric Endocrinology and Rheumatology, in the Department of Endocrinology, Metabolism and Internal Diseases at Poznan University of Medical Sciences and in the Department of Internal Medicine and Endocrinology, Medical University of Warsaw. Among these patients were 66 sporadic patients and nine familial cases of the disease (14 affected individuals in total). An average age at the moment of diagnosis was $9.2 \pm 6.1$ years. All adult patients at the time of the study were retested in adulthood. All of these patients suffered from $\mathrm{GH}$ deficiency, 98.8\% had gonadotropins and TSH deficiency, $32.5 \%$ had prolactin deficiency and $33.8 \%$ had ACTH deficiency. Pituitary Magnetic Resonance Imaging (MRI) was performed in all patients. Methods describing evaluation of hormonal status and pituitary imaging were described in our previous publication (4). In familial cases of the disease, no consanguinity was reported for any of the recruited families. All subjects were informed about the purpose of the study and their written consent was obtained. The Bioethical Committee of Poznan University of Medical Sciences approved the study. A population cohort of 104 individuals with WES data, and originating from the same region of Poland was used as a control group for excluding population specific variants while using WES in CPHD patients.

\section{GENETIC EXAMINATIONS}

\section{Targeted Sequencing of Pituitary Genes}

In order to search for mutations in known genes involved in pituitary organogenesis, patients were routinely screened using a targeted panel for point mutations in five pituitary genes. The bi-directional capillary sequencing was employed for PROP1, HESX, POU1F1, LHX3, and LHX4, genes. For all selected genes the coding sequences, together with $\sim 50$ nucleotides from neighboring intronic regions, were amplified from genomic DNA in independent PCR reactions [conditions were already published (5)]. Primers were designed using the Primer3 program (http://www.genome.wi.mit.edu/genome_software/ other/primer3.html/). The PCR products were visualized on a $2 \%$ agarose gel and purified using an Affymetrix ExoSAP-IT enzymatic PCR clean-up system. Sequencing reactions were performed using the BigDye Terminator kit (PE Biosystems, Rockville, USA) and subsequently visualized using a 3730xl DNA Analyzer (Applied Biosystems, Rockville, USA). The sequence tracks were further evaluated for quality metrics and subsequently examined for mutations with the use of CodonCode Aligner (http://www.codoncode.com), and reference sequence retrieved from GenBank (http://www.ncbi. nlm.nih.gov/genbank).

\section{Whole Exome Sequencing and Pathogenicity Prediction}

Patients with excluded mutations in PROP1, HESX, POU1F1, $L H X 3$, and $L H X 4$ genes were analyzed further using exome sequencing. A total of $1 \mu \mathrm{g}$ of genomic DNA from subjects was used for the construction of a library with the TruSeq DNA Sample Preparation Kit (Illumina). Whole exome enrichment was performed with the use of DNA library and TruSeq Exome Enrichment Kit (Illumina). The achieved assay performance was as follows: The minimal mean depth of target regions was $218 \mathrm{x}$ (post-alignment) and the average read length 149 bp. The percentage of bases in target regions with a depth of coverage $>20 \mathrm{X}-98.6 \%,>30 \mathrm{X}-97.5 \%,>50 \mathrm{x}-94.1 \%$. Ninetynine percentage of the reads was aligned and mapped to human genome reference sequence hg19 (BWA v0.7.12, Picard v1.130, GATK v3.4.0, SnpEff v4.1g) of which $90.5 \%$ were nonredundant. For pathogenicity evaluation, the following features were applied: (a) gene/transcript annotations (downloaded from UCSC GenomeBrowser, hg19), (b) known sequence variants from dbSNP (version 142), 1,000 Genomes project (The 1,000 Genomes Project Consortium, Phase3), ExAc Database (http:// exac.broadinstitute.org/) and GenomAD v2.1.1 (6). Selected alterations, which were found using NGS were confirmed using 
Sanger sequencing and no discrepancy between these results was detected. The identified variants were analyzed for their impact on protein structure and functionality and pathogenicity estimation using following algorithms: Sift (7), and PolyPhen2 (8), dbNSFP (9), FATHMM (10), MutationTaster v2 (11), PhenIX (12), CADD (13), and HPO database (14) as well as population datasets. Nucleotide sequences (coding and neighboring $\sim 50$ nt intronic) were explored for Splice Site disturbances using Human Splicing Finder (HSF3.0) (15) and NNsplice v. 0.9 (16). The variant prioritization was based on the variant frequency (novel or very rare variants), functional prediction using selected algorithms (VEP tool, Ensembl), and possible contribution of a genes into pituitary functioning (HPO) (17). All variants were classified according ACMG recommendations (18) as presented in Table 2, with the use of InterVar tool (19) and Varsome (20), checked individually for correctness of estimation and adjusted manually if needed. Prediction of protein conformation changes was accomplished using Phyre2 (21) and UCSF Chimera 1.7 (22).

\section{RESULTS}

Among examined CPHD patients a subtle predominance of males $(43 \mathrm{M} / 37 \mathrm{~F})$ was noted that is in accordance with published epidemiologic data (23-25). Abnormalities in pituitary morphology were observed in majority of patients (95\%). Pituitary Stalk Interruption Syndrome (PSIS) was diagnosed in 12 patients $(22.7 \%)$. The analysis of major CPHD transcription factors resulted in identification of mutations in all nine familial cases (14 patients) and 32 sporadic patients (45\% of all patients), all without PSIS. Mutations were found only in PROP1 gene and they represented recessive founder 2-bp deletion c.301_302delAG (p.L102Cfs*8) and c.150delA (p.R53Dfs*112). Among these mutations, homozygosity for c.301_302delAG was detected in 11 cases (30\% of patients affected by PROP1 mutation), homozygosity for c.150delA in 10 patients (27\%) and 15 cases presenting compound heterozygosity (41\%). No abnormalities were identified in HESX, POU1F1, LHX3, and LHX4 genes in the coding sequences. Neither structural rearrangements within those genes, nor abnormalities in particular exon copy number were detected using MLPA (unpublished data). Clinical characteristics of the patients regarding their genetic status was reported in Table $\mathbf{1}$.

\section{Whole Exome Sequencing in PROP1 Negative Patients}

All of the patients that did not reveal any abnormalities in conventional sequencing of the five most common CPHD genes were subjected for whole exome sequencing (WES). In two cases, likely pathogenic (according to ACMG criteria) and protein damaging variants affecting IGSF10 and SEMA3A genes were identified. For the IGSF10 gene a novel variant c.5014G >A: p.A1672T was found (Case 1). Analysis of relatives revealed de novo origin of this alteration. In another patient presenting CPHD with PSIS (Case 3), a unique likely pathogenic, homozygous SEMA3A allele carrying two variants: c.1302_1303delinsCA (p.V435delinsI) and c.1453-9delC was

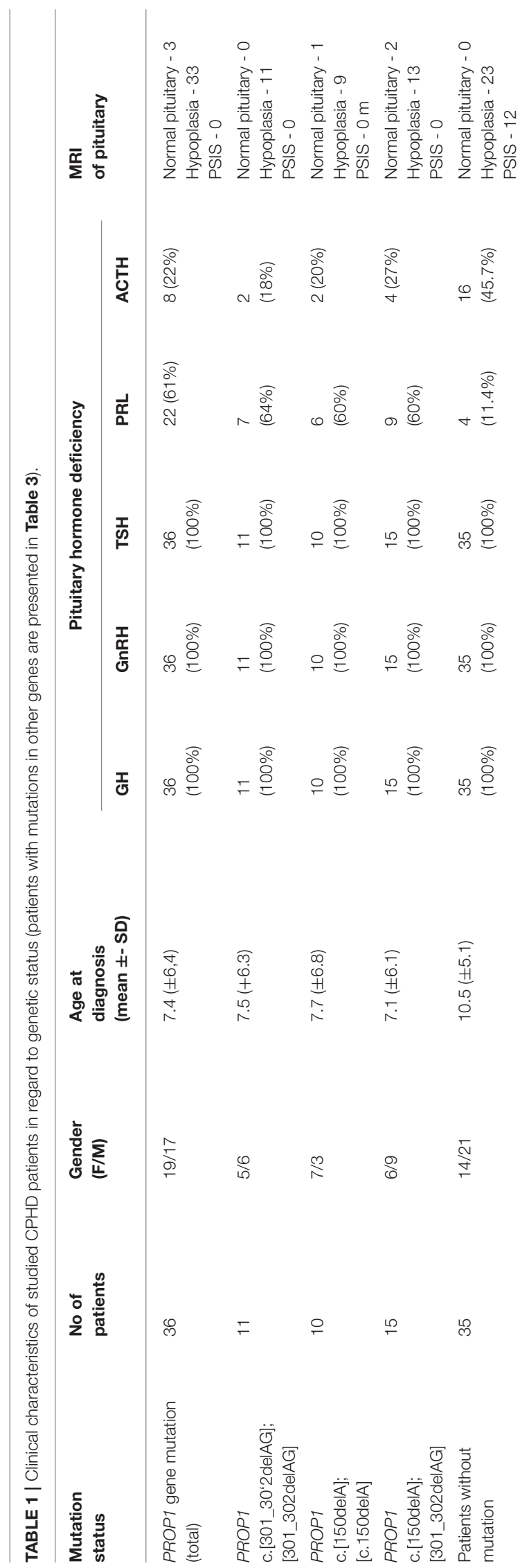


TABLE 2 | Exome sequencing results of studied patients with CPHD including variant description, effect on protein, allele frequency, classification and pathogenicity prediction.

Variants predicted to affect protein function (pathogenic/VUS according to ACMG criteria)

Case Gene Chrom Variant description to Acfect on

Effect on dbSNP

Mode of

ACMG variant $\quad$ ACMG

MAF CADD Score Protein function

Position

HGVS, nucleotide

protein

(rs number)

inheritance classification

gnomAD PHRED/RAW

prediction

IGSF10 3:151162755 p.A1672T, c.5014G>A, NM_178822.4 het.

Damaging,

Novel

Unknown (AD Likely

postulated) pathogenic

PS2, PM1,

change

2 IGSF10 3:151154509 p.D2614N c.7840G>A, Damaging NM_178822.4 het. conformation

rs112889898

Unknown (AD VUS/Benign

PM $2, \mathrm{PP}$,

change

GLI2 2:121748048 p.D1520N(:)M1352V Probably

2:121747544 c. $4558 G>A(:) c .4054 A>G$ damaging

\section{rs114814747} postulated) comp.het

3 SEMA3A 7:83634712- p.V435delinsl,

Damaging,

conformati

Nove

VUS/Benig

c.1302_1303delinsCA

change,

7:83614801- C.1453-9del C

Splicing affected, rs141423527 acceptor gained

\section{Variants predicted to affect splicing (VUS/benign according to ACMG criteria)}

$4 \quad$ IGSF10 $3: 151161101 \quad$ p.L1878L, Synonymous SNV, Novel

c. $5634 \mathrm{C}>\mathrm{G}$,

splicing affected,

NM_178822.4 het.

activation of an

exonic cryptic

donor site

OTX2 14:57268750 p.G199G

c. $597 \mathrm{C}>\mathrm{G}$,

Synonymous SNV, Novel

splicing affected,

NM_001270525.1, het. activation of an

exonic cryptic

donor site

5 SEMA3A 7:83764113 p.Q89Q,

c. $267 \mathrm{~A}>\mathrm{G}$

NM_006080 het.

Synonymous SNV, rs74349534

splicing affected,

donor lost

PCSK1 5:95757703 c.544-43T>G

NM 000439.4

Splicing affected, Novel

acceptor gained

$A D$

Lik

PM1, PM2,

pathogenic

PM6, PP4,

$A D$

Benign

PP3, PP4

Unknown (AD VUS

postulated) Uncertan

BP4, BP7,

$A D$

Benign

PM2, PM6,

PP4, BP4,

BP7

Synonymous SNV, Novel

splicing affected,

enhancer site

broken

PP4, BS1,

BS2, BP4,

BP7,

PM6, PP4,

BS1, BS2,

BP4, BP7,

$A D$

VUS Uncertain

PS4, PM2,

PM6, PP4,

BP4, BP7,
33/6.91 Control of early migration of neurons expressing gonadotropin-releasing hormone (GnRH neurons)

28.5/6.15 As above

32/4.80 Transcription factor which bind DNA

7.8/0.55 through zinc finger motifs, mediator of Sonic hedgehog (Shh) signaling

26.6/3.82 Chemorepulsive factor, inhibiting axonal outgrowth, and

chemoattractive agent, stimulating the growth of apical dendrites

$10.74 / 0.65$

5.84/0.20 Control of early migration of neurons expressing gonadotropin-releasing hormone (GnRH neurons)

20.8/2.14 Transcription factor that plays a role in brain, craniofacial, and sensory organ development. Mutations in

this gene cause syndromic

microphthalmia 5 (MCOPS5) and combined pituitary hormone deficiency 6 (CPHD6)

0.009

15.94/1.53 Chemorepulsive factor, inhibiting axonal outgrowth, and

chemoattractive agent, stimulating the growth of apical dendrites

1.74/0.002 Proteolytic activation of polypeptide hormones and neuropeptides precursors

5.78/1.51 Transcription regulator, commonly mutated in hypogonadotropic hypogonadism patients 
TABLE 2 | Continued

Variants predicted to affect protein function (pathogenic/VUS according to ACMG criteria)

Chrom. Variant description

Effect on

dbSNP

Mode of

ACMG variant

ACMG

Position HGVS, nucleotide,

protein

(rs number)

inheritance classification

evidence

MAF

CADD Score Protein function

prediction

v2.1.1

$7 \quad$ WDR11 10:122622238 c.527-9T>C

Intron, splicing

Novel

$A D$

$A D$

Benign

PM6, PP4,

affected acceptor

BS1, BS2,

gained

BP4, BP7,

$8 \quad$ FGF17 8:21900402 $\quad$ c.-38delG

NM_003867.3 het.

5'UTR, splicing

rs147561706

$A D$

Benign

PM6, PP4,

affected, donor

$\mathrm{BS} 1, \mathrm{BS} 2$

gained

BP4, BP7,

0.009

olved in a variety of cellular processes, including cell cycle progression, signal transduction, apoptosis, and gene regulation

FGF8 10:103530438 c.445-62G>A

Splicing affected, rs3218238

AD

Benign

PM6, PP4,
BS1, BS2,

BS1, BS2
BP4, BP7

0.02

acceptor gained

4, BP7

normal brain development.

Mutations in this gene are the cause of hypogonadotropic

hypogonadism 20 with or without

$$
\text { anosmia }
$$

Synonymous SNV, rs61732852
splicing affected,
activation of an
exonic cryptic
acceptor site,
with presence of
one or more
cryptic branch
points

AD

Likely Benign

PM2, BP4,

$\mathrm{BP} 7$,

0.004

9 GLI2 2:121742124
N.T587T; c.1761G $>$ 005270.4, het.

splicing affected,

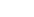

Variety of biological processes, including embryonic development, cell growth, morphogenesis, tissue repair. Supports androgen and anchorage independent growth of mammary tumor cells

exonic cryptic

with presence of

points 

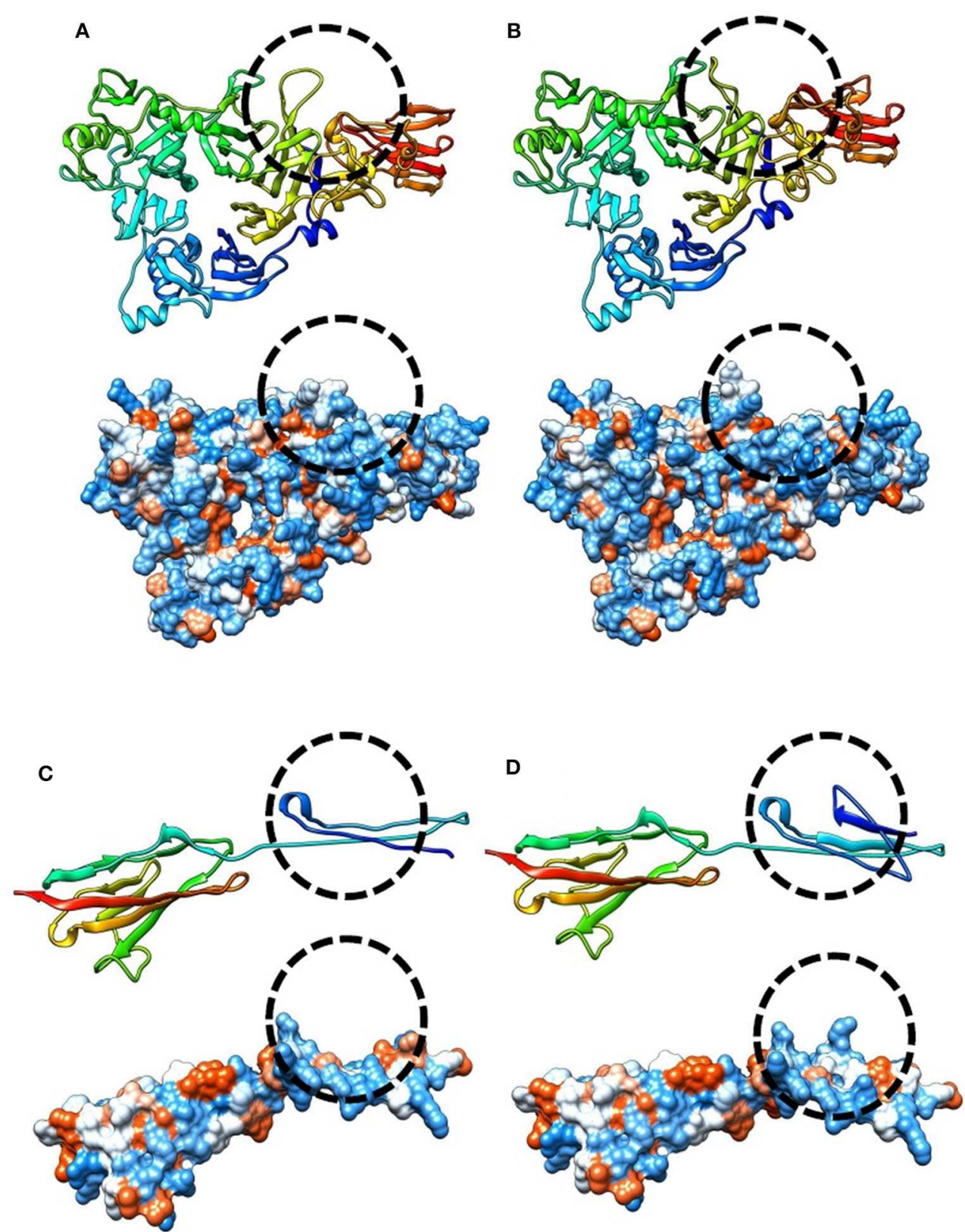

FIGURE 1 | Modeling and prediction of conformational changes of SEMA3A and IGSF10 variants. Predicted conformation changes surrounding variant position are highlighted and encircled using dashed line. (A) Ribbon (top) and hydrophobic (bottom) model of reference SEMA3A protein sequence, (B) Ribbon (top) and hydrophobic (bottom) prediction of SEMA3A p.V435I variant. (C) Ribbon (top) and hydrophobic (bottom) model of reference IGSF10 sequence covering a third lg-like C2-type domain (1648-1739AA), (D) Ribbon (top) and hydrophobic (bottom) prediction of p.A1672T variant affecting third Ig-like C2-type domain of IGSF10 protein.

detected. The first reported variant c.1302_1303delinsCA is novel and likely pathogenic, whereas the second is rare (MAF 0.01 ) and therefore considered as benign with a rather minor impact. For these two patients and their detected variants, all used pathogenicity prediction algorithms consistently showed deleterious effect (Table 2, Figure 1), supporting causativeness.

Except for these two cases, less convincing evidence and clinical relevancy was found for the detected variants in another seven patients (classified as variants of unknown significance VUS or benign according to ACMG criteria). For Case 2 (the patient presenting CPHD with PSIS), the damaging prediction was established for c.7840G $>$ A (p.D2614N; rs112889898) of IGSF10 and the two coexisting variants in CPHD gene GLI2 (rs114814747, rs149140724). However, these changes were considered as VUS, since they were reported in population databases. In another patient (Case 4), synonymous 
TABLE 3 | Clinical characteristics of studied CPHD patients with genetic defects in SEMA3A, IGSF10, PCSK1, and IHH genes.

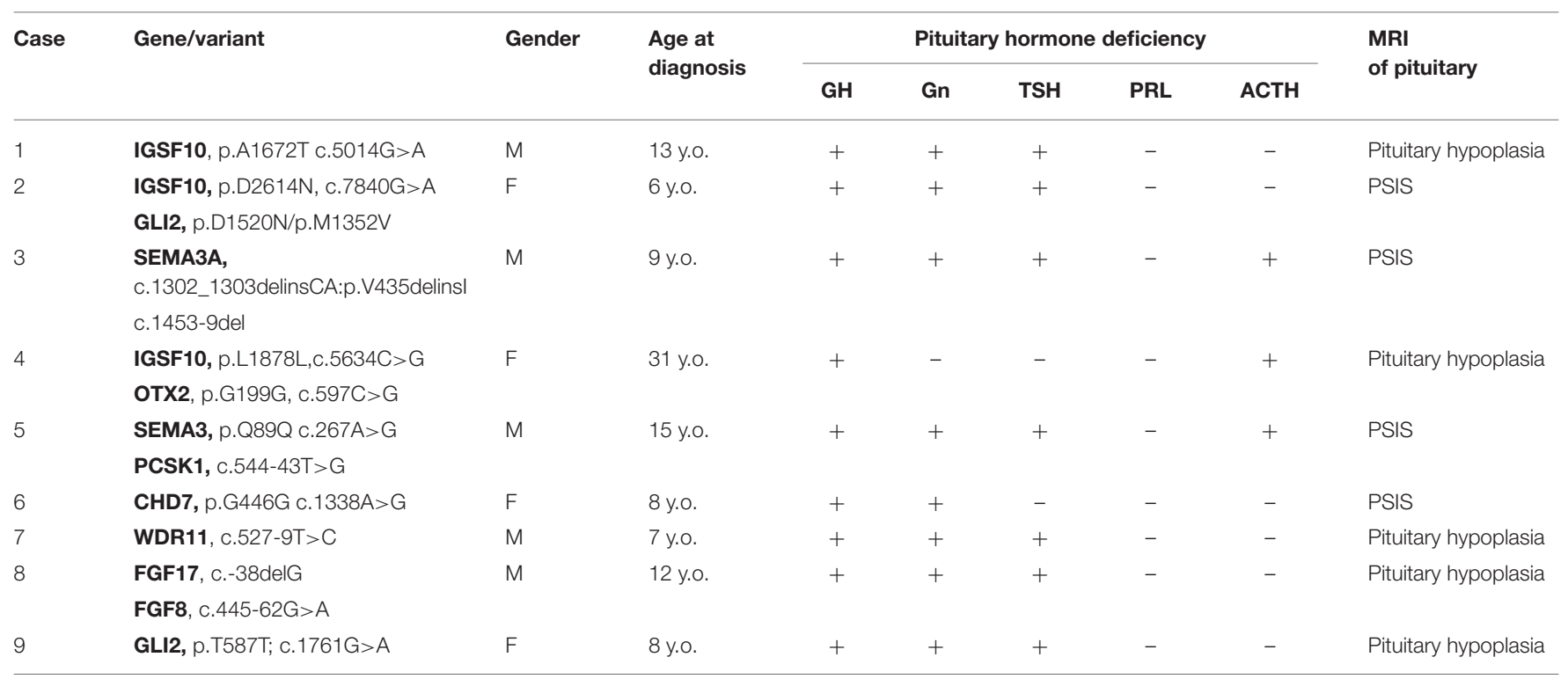

In all reported patients with new genetic defects, no other clinical abnormalities were reported.

change in IGSF10 (c.5634C>G; p.L1878L) co-occurred with alteration in OTX2 (c.597C>G; p.G199G), another gene with evidenced contribution to CPHD. These changes represent novel unreported variants, but only splice-site algorithms supported estimation of pathogenicity and therefore further functional experiments are required. Pathogenic, disease-causing splicesite predictions were also found for another five variants. In SEMA3A gene, a splice variant c.267A $>$ G (p.Q89Q) was found in combination with heterozygous intronic change in the PCSK1 gene (c.544-43T>G). Novel variants in two genes linked to hypogonadotropic hypogonadism: $C H D 7$ (c.1338A $>\mathrm{G}$, p.G446G) and WDR (c.527-9T >C, intronic) were identified in the next two patients. In total four out of nine cases we noted co-occurrence of predicted deleterious variants in two different genes, supporting growing recent evidence on possible oligogenic background of CPHD. All changes with their genomic coordinates and pathogenicity prediction were included in Table 2. All of the novel variants were screened in the population cohort (104 controls, 208 alleles) and were absent. The clinical characteristics of patients affected by mutations in genes excluding $P R O P 1$, are presented in Table 3 . It is noteworthy that four of the detected mutations were identified in subjects presenting CPHD with PSIS and five other CPHD without PSIS. The protein conformation prediction for IGSF10 p.A1672T variant (Case 1) and SEMA3A p.V435delinsI variant (Case 3) are shown in Figure 1. Pedigrees for both cases are presented in Figure 2.

\section{DISCUSSION}

In this study we examined 80 CPHD patients (nine families, 66 sporadic cases), searching for genetic abnormalities. Causative mutations were found in the PROP1 transcription factor gene

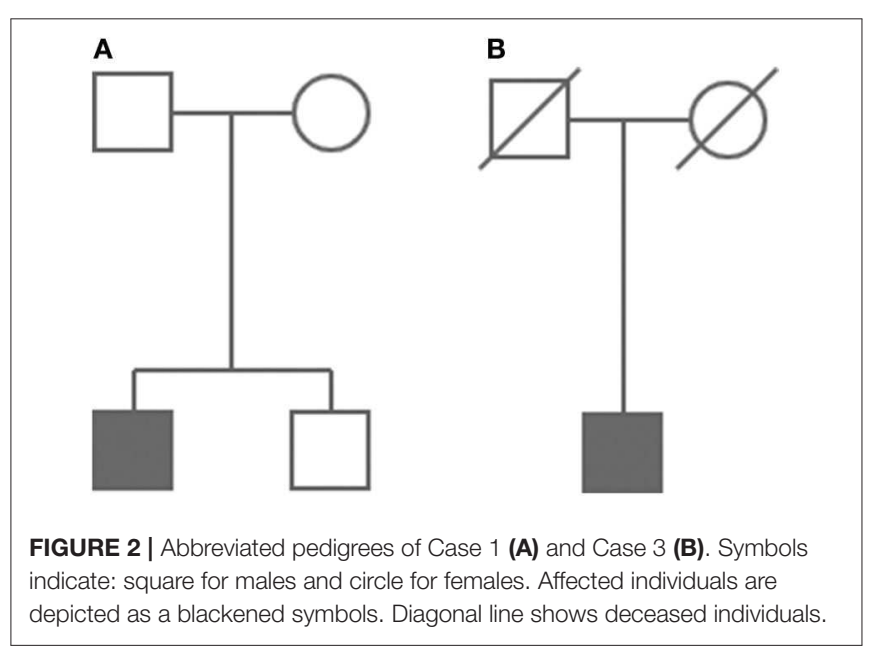

as well as two other genes known to contribute to isolated hypogonadotropic hypogonadism (IHH), namely IGSF10 and $S E M A 3 A$. Our results are in agreement with multiple studies reporting the occurrence of common PROP1 founder mutations in sporadic and familial cases (26-29). The most frequent mutation in our cohort was the 2-bp deletion c.301_302delAG (p.Leu102Cysfs*8), detected in 26 cases (11 homozygotes and 15 compound heterozygotes). This frameshift deletion is the most common PROP1 mutation and leads to a premature stop codon in the residue 110 . The second most common PROP1 variant was 1-bp deletion c.150delA (p.Arg53Aspfs*112) found in 25 cases (10 homozygotes and 15 compound heterozygotes). The high frequency of both mutations is caused due to the presence of two ancestral founder variants - c.301_302delAG originated from the Baltic Sea area around 2,525 years ago 


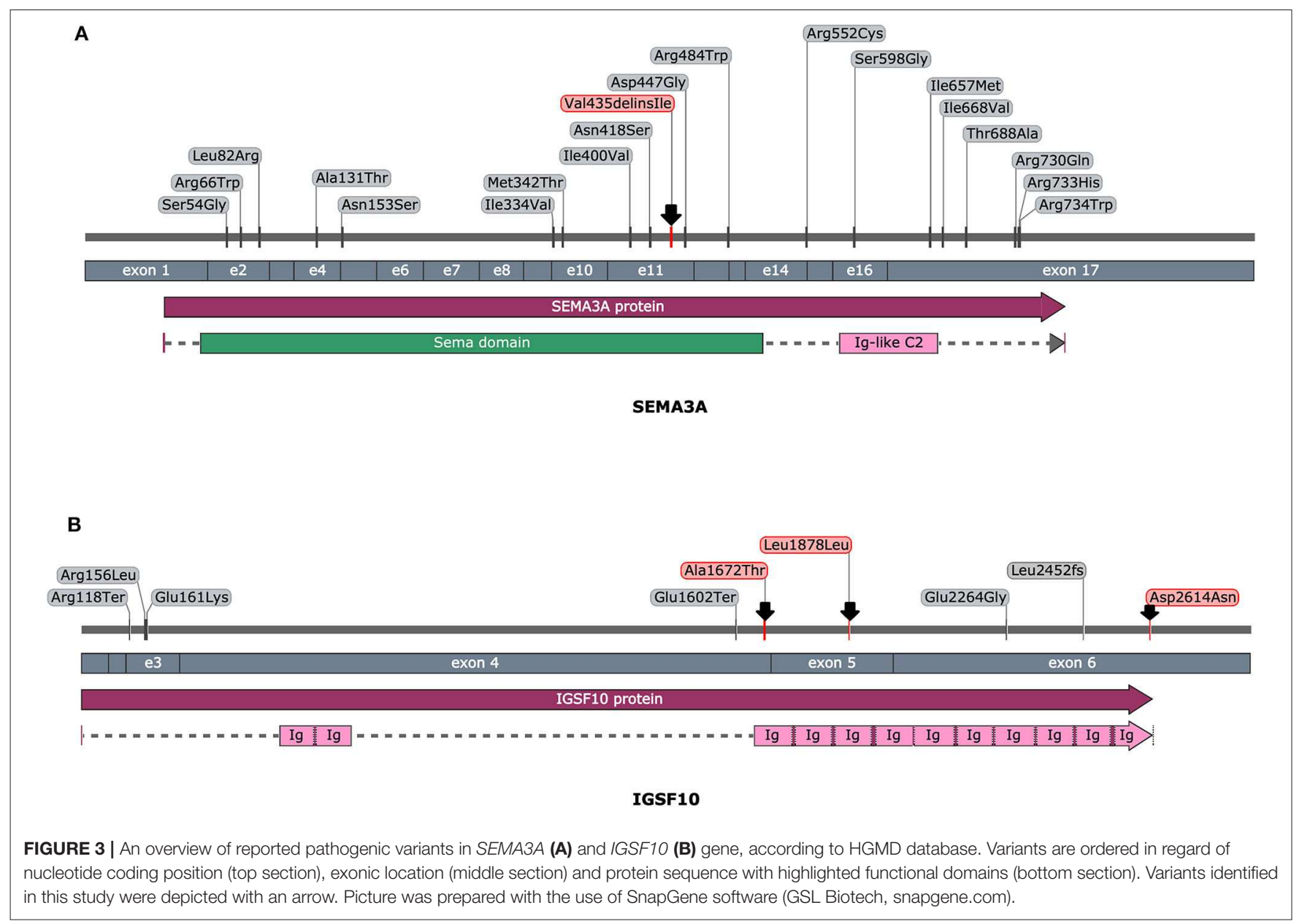

and c.150delA from the Belarus region around 1,093 years ago $(30,31)$. There were no other changes reported for this gene, which is not surprising since other mutations are ultrarare and contribute to a minority of patients $(5,32)$. The other disruptive variants affecting POU1F1, LHX3, and LHX4, contribute only occasionally to the pituitary phenotype (33-35). In this study, we also detected likely pathogenic occurrence of point mutations disturbing the SEMA3A and IGSF10 genes, so far not linked to CPHD. The semaphorin 3A gene (SEMA3A) is a member of the semaphorin family and encodes a protein composed of immunoglobulin-like domain (C2-type), a PSI domain and major Sema domain. This protein shows activity as either a chemorepulsive agent, inhibiting axonal outgrowth, or as a chemoattractive agent, stimulating the growth of apical dendrites (36). In both cases, the protein is vital for normal neuronal pattern development. Semaphorin 3 induces the collapse and paralysis of neuronal growth cones and serves as a ligand that guides specific growth cones by a motilityinhibiting mechanism (37). The protein is therefore involved in the development of the olfactory system and in neuronal control during puberty. Due to SEMA3A genes' pivotal role in development, the gene was widely examined for contribution to various human disorders. Heterozygous variants have been associated with hypogonadotropic hypogonadism and anosmia (Kallmann syndrome, OMIM \# 614897, autosomal dominant inheritance) (38-41). In another report Hofmann et al., presented biallelic SEMA3A loss of function mutations causing syndromic short stature (42). The phenotype of the reported patient resembles knock-out mice symptoms (relative macrocephaly, camptodactyly, septal heart defect) indicating a novel autosomal recessive type of syndromic short stature without obvious signs of Kallmann syndrome. In this report, we found for the first time SEMA3A allele bearing two variants (c.1302_1303delinsCA, c.1453-9delC) in the homozygous state, thus indicating a novel attractive target contributing to pituitary organogenesis. The identified variant is supporting evidence of recessive inheritance and milder outcome comparing to previously reported conditions. Another likely pathogenic variant was found in the IGSF10 gene, which is a player recently evidenced to be causative for isolated hypogonadotropic hypogonadism and delayed puberty. Immunoglobulin Superfamily Member 10 (IGSF10) is a component of a complex system of migratory cues guiding GnRH neurons from their origin in the nasal placode toward the hypothalamus during embryogenesis. Howard et al. showed that IGSF10 mutations dysregulate gonadotropinreleasing hormone neuronal migration (43). Using exome 
and candidate gene sequencing, the authors identified rare heterozygous mutations in IGSF10 in six unrelated families, which resulted in intracellular retention with failure in the secretion of mutant proteins and self-limited delayed puberty segregated within families, displaying an autosomal dominant pattern of inheritance. A pathogenic variant in IGSF10 was found in one of our CPHD patients. Another two patients presented with VUS/benign IGSF10 alterations that co-occurred with a variant in the CPHD gene (GLI2 or OTX2) and, therefore, its true contribution to the disease is unclear. In addition to this, we also noted that all reported IGSF10 variants affect highly conserved residues among homologs, as revealed by PhyloP (an overview presenting reported mutations in IGSF10 and SEMA3A was shown in Figure 3). A recent report presenting WES examination in pituitary stalk interruption syndrome (PSIS), was published by Zwaveling-Soonwala et.al. (44). Authors indicated a particularly common contribution of rare GLI2 variants for PSIS patients (in total 16 were reported). We identified GLI2 rare change only for one out of 12 cases with PSIS - showing combined p.D1520N/p.M1352V changes (cooccurring with IGSF10 p.D2614N) and in the other patient with pituitary hypoplasia without PSIS (a benign synonymous SNV p.T587T as well as predicted activation of the exonic cryptic splice-site). In our cohort of patients, however, we could not confirm presence of cadherin gene DCHS1 defects frequently mutated in PSIS patients. It is noteworthy that among PSIS cases two other benign variants, in IHH genes CHD7 and FGF8, were also identified (both predicted to affect splicing). These findings may support the growing role of $\mathrm{IHH}$ genes in etiopathogenesis of CPHD. In another study Simm et al. (45) reported two new genes contributing to CPHD, SLC20A1, and SLC15A4 and evidenced that a significant portion of cases arise as a de novo event. Another study utilizing NGS sequencing also shed a new light on the combination of rare variants in different genes that might explain the incomplete penetrance in CPHD and the complexity of the disorder $(46,47)$. In conclusion, we confirmed the major contribution of PROP1 gene mutations in CPHD. The analysis indicated founder deleterious variants as the most frequent changes present but no novel mutations were found. No other convincingly pathogenic changes were identified in other known CPHD transcription factors. Only the GLI2 combined variant (p.D1520N/p.M1352V) was found in one patient which is seemingly more frequent among CPHD patients, as reported

\section{REFERENCES}

1. Fang Q, George AS, Brinkmeier ML, Mortensen AH, Gergics P, Cheung LY, et al. Genetics of combined pituitary hormone deficiency: roadmap into the genome era. Endocr Rev. (2016) 37:636-75. doi: 10.1210/er.2016-1101

2. Kelberman D, Rizzoti K, Lovell-Badge R, Robinson IC, Dattani MT. Genetic regulation of pituitary gland development in human and mouse. Endocr Rev. (2009) 30:790-829. doi: 10.1210/er.2009-0008

3. Stamou MI, Cox KH, Crowley WFJr. Discovering genes essential to the hypothalamic regulation of human reproduction using a human disease model: adjusting to life in the "-omics" era. Endocr Rev. (2015) 36:603-21. doi: 10.1210/er.2015-1045 by Zwaveling-Soonwala et al. (44). Despite this, we identified a single pathogenic mutation in the SEMA3A, and IGSF10 genes producing two new candidates potentially causative for CPHD etiology. Both genes are coding proteins that are involved in neuronal growth and migration, therefore potentially influencing proper pituitary development.

\section{DATA AVAILABILITY STATEMENT}

The datasets generated for this study can be found in the Department of Endocrinology, Metabolism and Internal Diseases, Poznan University of Medical Sciences, Poznan, Poland.

\section{ETHICS STATEMENT}

The studies involving human participants were reviewed and approved by The Bioethical Committee of Poznan University of Medical Sciences. The patients/participants provided their written informed consent to participate in this study.

\section{AUTHOR CONTRIBUTIONS}

$\mathrm{BB}$ and $\mathrm{KZ}$ contributed to conception and design of the study. $\mathrm{BB}, \mathrm{TZ}, \mathrm{EB}-\mathrm{O}$, and $\mathrm{AJ}$ contributed to genomic analyses. KZ, MK, PG, MN, MO-M, BR-P, KK-K, MS, UA, TB, and MR performed clinical examinations. EW participate in lab work. $\mathrm{BB}$ and $\mathrm{KZ}$ wrote the first draft of the manuscript. All authors contributed to manuscript revision, read, and approved the submitted version.

\section{FUNDING}

This work was supported by the grant no. P05B 10825 from The Polish State Committee for Scientific Research at the Ministry of Science and Higher Education and by The Polish National Science Center grant DEC-2012/04/M/NZ5/00475.

\section{ACKNOWLEDGMENTS}

We would like to thank all patients for their contribution to the study. We also would like to thank Mr. Maximillian Atkinson for his time spent on revision of our manuscript and improvement. 
7. Kumar P, Henikoff S, Ng PC. Predicting the effects of coding non-synonymous variants on protein function using the SIFT algorithm. Nat Protoc. (2009) 4:1073-82. doi: 10.1038/nprot.2009.86

8. Adzhubei IA, Schmidt S, Peshkin L, Ramensky VE, Gerasimova A, Bork P, et al. A method and server for predicting damaging missense mutations. Nat Methods. (2010) 7:248-9. doi: 10.1038/nmeth0410-248

9. Liu X, Wu C, Li C, Boerwinkle E. dbNSFP v3.0: a one-stop database of functional predictions and annotations for human nonsynonymous and splice-site SNVs. Hum Mutat. (2016) 37:235-41. doi: 10.1002/humu.22932

10. Shihab HA, Gough J, Cooper DN, Stenson PD, Barker GL, Edwards KJ, et al. Predicting the functional, molecular, and phenotypic consequences of amino acid substitutions using hidden Markov models. Hum Mutat. (2013) 34:57-65. doi: 10.1002/humu.22225

11. Schwarz JM, Cooper DN, Schuelke M, Seelow D. MutationTaster2: mutation prediction for the deep-sequencing age. Nat Methods. (2014) 11:361-2. doi: $10.1038 /$ nmeth. 2890

12. Zemojtel T, Kohler S, Mackenroth L, Jager M, Hecht J, Krawitz P, et al. Effective diagnosis of genetic disease by computational phenotype analysis of the disease-associated genome. Sci Transl Med. (2014) 6:252ra123. doi: 10.1126/scitranslmed.3009262

13. Rentzsch P, Witten D, Cooper GM, Shendure J, Kircher M. CADD: predicting the deleteriousness of variants throughout the human genome. Nucleic Acids Res. (2019) 47:D886-94. doi: 10.1093/nar/gky1016

14. Kohler S, Vasilevsky NA, Engelstad M, Foster E, Mcmurry J, Ayme S, et al. The human phenotype ontology in 2017. Nucleic Acids Res. (2017) 45:D865-76. doi: $10.1093 /$ nar/gkw1039

15. Desmet FO, Hamroun D, Lalande M, Collod-Beroud G, Claustres M, Beroud C. Human Splicing Finder: an online bioinformatics tool to predict splicing signals. Nucleic Acids Res. (2009) 37:67-8. doi: 10.1093/nar/gkp215

16. Reese MG, Eeckman FH, Kulp D, Haussler D. Improved splice site detection in Genie. J Comput Biol. (1997) 4:311-23. doi: 10.1089/cmb.1997.4.311

17. Jalali Sefid Dashti M, Gamieldien J. A practical guide to filtering and prioritizing genetic variants. Biotechniques. (2017) 62:18-30. doi: $10.2144 / 000114492$

18. Richards S, Aziz N, Bale S, Bick D, Das S, Gastier-Foster J, et al. Standards and guidelines for the interpretation of sequence variants: a joint consensus recommendation of the American College of Medical Genetics and Genomics and the Association for Molecular Pathology. Genet Med. (2015) 17:405-24. doi: $10.1038 /$ gim. 2015.30

19. Li Q, Wang K. InterVar: clinical interpretation of genetic variants by the 2015 ACMG-AMP guidelines. Am J Hum Genet. (2017) 100:267-80. doi: 10.1016/j.ajhg.2017.01.004

20. Kopanos C, Tsiolkas V, Kouris A, Chapple CE, Albarca Aguilera M, Meyer $\mathrm{R}$, et al. VarSome: the human genomic variant search engine. Bioinformatics. (2019) 35:1978-80. doi: 10.1093/bioinformatics/bty897

21. Kelley LA, Mezulis S, Yates CM, Wass MN, Sternberg MJE. The Phyre2 web portal for protein modeling, prediction and analysis. Nat Protoc. (2015) 10:845. doi: 10.1038/nprot.2015.053

22. Pettersen EF, Goddard TD, Huang CC, Couch GS, Greenblatt DM, Meng EC, et al. UCSF Chimera-a visualization system for exploratory research and analysis. J Comput Chem. (2004) 25:1605-12. doi: 10.1002/jcc. 20084

23. Lamberts SW, De Herder WW, Van Der Lely AJ. Pituitary insufficiency. Lancet. (1998) 352:127-34. doi: 10.1016/S0140-6736(98)02254-5

24. Ascoli P, Cavagnini F. Hypopituitarism. Pituitary. (2006) 9:335-42. doi: 10.1007/s11102-006-0416-5

25. Kelberman D, Dattani MT. The role of transcription factors implicated in anterior pituitary development in the aetiology of congenital hypopituitarism. Ann Med. (2006) 38:560-77. doi: 10.1080/07853890600994963

26. Cogan JD, Wu W, Phillips JA III, Arnhold IJ, Agapito A, Fofanova OV, Osorio MG, et al. The PROP1 2-base pair deletion is a common cause of combined pituitary hormone deficiency. J Clin Endocrinol Metab. (1998) 83:3346-9. doi: $10.1210 /$ jc. 83.9 .3346

27. Fluck C, Deladoey J, Rutishauser K, Eble A, Marti U, Wu W, et al. Phenotypic variability in familial combined pituitary hormone deficiency caused by a PROP1 gene mutation resulting in the substitution of Arg$>$ Cys at codon 120 (R120C). J Clin Endocrinol Metab. (1998) 83:3727-34. doi: $10.1210 /$ jc. 83.10 .3727
28. Wu W, Cogan JD, Pfaffle RW, Dasen JS, Frisch H, O'connell SM, et al. Mutations in PROP1 cause familial combined pituitary hormone deficiency. Nat Genet. (1998) 18:147-9. doi: 10.1038/ng0298-147

29. Deladoey J, Fluck C, Buyukgebiz A, Kuhlmann BV, Eble A, Hindmarsh PC, et al. "Hot spot" in the PROP1 gene responsible for combined pituitary hormone deficiency. J Clin Endocrinol Metab. (1999) 84:1645-50. doi: $10.1210 / j$ c.84.5.1645

30. Krzisnik C, Grguric S, Cvijovic K, Laron Z. Longevity of the hypopituitary patients from the island Krk: a follow-up study. Pediatr Endocrinol Rev. (2010) 7:357-62.

31. Dusatkova P, Pfaffle R, Brown MR, Akulevich N, Arnhold IJ, Kalina MA, et al. Genesis of two most prevalent PROP1 gene variants causing combined pituitary hormone deficiency in 21 populations. Eur J Hum Genet. (2016) 24:415-20. doi: 10.1038/ejhg.2015.126

32. Penta L, Bizzarri C, Panichi M, Novelli A, Lepri FR, Cappa M, et al. Identification of a novel PROP1 mutation in a patient with combined pituitary hormone deficiency and enlarged pituitary. Int J Mol Sci. (2019) 20:1875. doi: 10.3390/ijms20081875

33. Dateki S, Fukami M, Uematsu A, Kaji M, Iso M, Ono M, et al. Mutation and gene copy number analyses of six pituitary transcription factor genes in 71 patients with combined pituitary hormone deficiency: identification of a single patient with LHX4 deletion. J Clin Endocrinol Metab. (2010) 95:4043-7. doi: $10.1210 /$ jc. $2010-0150$

34. Gucev Z, Tasic V, Plaseska-Karanfilska D, Konstantinova MK, Stamatova A, Dimishkovska M, et al. LHX4 Gene alterations: patient report and review of the literature. Pediatr Endocrinol Rev. (2016) 13:749-55.

35. Cohen E, Maghnie M, Collot N, Leger J, Dastot F, Polak M, et al. Contribution of LHX4 mutations to pituitary deficits in a cohort of 417 unrelated patients. J Clin Endocrinol Metab. (2017) 102:290-301. doi: 10.1210/jc.201 6-3158

36. Polleux F, Morrow T, Ghosh A. Semaphorin 3A is a chemoattractant for cortical apical dendrites. Nature. (2000) 404:567-73. doi: 10.1038/350 07001

37. Wu KY, Hengst U, Cox LJ, Macosko EZ, Jeromin A, Urquhart ER, et al. Local translation of RhoA regulates growth cone collapse. Nature. (2005) 436:1020-4. doi: 10.1038/nature03885

38. Cariboni A, Davidson K, Rakic S, Maggi R, Parnavelas JG, Ruhrberg C. Defective gonadotropin-releasing hormone neuron migration in mice lacking SEMA3A signalling through NRP1 and NRP2: implications for the aetiology of hypogonadotropic hypogonadism. Hum Mol Genet. (2011) 20:336-44. doi: $10.1093 / \mathrm{hmg} / \mathrm{ddq} 468$

39. Hanchate NK, Giacobini P, Lhuillier P, Parkash J, Espy C, Fouveaut C, et al. SEMA3A, a gene involved in axonal pathfinding, is mutated in patients with Kallmann syndrome. PLoS Genet. (2012) 8:e1002896. doi: 10.1371/journal.pgen.1002896

40. Young J, Metay C, Bouligand J, Tou B, Francou B, Maione L, et al. SEMA3A deletion in a family with Kallmann syndrome validates the role of semaphorin $3 \mathrm{~A}$ in human puberty and olfactory system development. Hum Reprod. (2012) 27:1460-5. doi: 10.1093/humrep/ des022

41. Kansakoski J, Fagerholm R, Laitinen EM, Vaaralahti K, Hackman P, Pitteloud $\mathrm{N}$, et al. Mutation screening of SEMA3A and SEMA7A in patients with congenital hypogonadotropic hypogonadism. Pediatr Res. (2014) 75:641-4. doi: $10.1038 /$ pr.2014.23

42. Hofmann K, Zweier M, Sticht H, Zweier C, Wittmann W, Hoyer J, et al. Biallelic SEMA3A defects cause a novel type of syndromic short stature. Am J Med Genet A. (2013) 161A:2880-9. doi: 10.1002/ajmg.a. 36250

43. Howard SR, Guasti L, Ruiz-Babot G, Mancini A, David A, Storr HL, et al. IGSF10 mutations dysregulate gonadotropinreleasing hormone neuronal migration resulting in delayed puberty. EMBO Mol Med. (2016) 8:626-42. doi: 10.15252/emmm.2016 06250

44. Zwaveling-Soonawala N, Alders M, Jongejan A, Kovacic L, Duijkers FA, Maas SM, et al. Clues for polygenic inheritance of pituitary stalk interruption syndrome from exome sequencing in 20 patients. J Clin Endocrinol Metab. (2018) 103:415-28. doi: 10.1210/jc.201701660 
45. Simm F, Griesbeck A, Choukair D, Weiss B, Paramasivam N, Klammt J, et al. Identification of SLC20A1 and SLC15A4 among other genes as potential risk factors for combined pituitary hormone deficiency. Genet Med. (2018) 20:728-36. doi: 10.1038/gim.2017.165

46. Guo QH, Wang CZ, Wu ZQ, Qin Y, Han BY, Wang AP, et al. Multi-genic pattern found in rare type of hypopituitarism: a whole-exome sequencing study of Han Chinese with pituitary stalk interruption syndrome. J Cell Mol Med. (2017) 21:3626-32. doi: $10.1111 / \mathrm{jcmm} .13272$

47. Rodriguez-Contreras FJ, Marban-Calzon M, Vallespin E, Del Pozo A, SolisLopez M, Lobato-Vidal N, et al. Loss of function BMP4 mutation supports the implication of the BMP/TGF-beta pathway in the etiology of combined pituitary hormone deficiency. Am J Med Genet A. (2019) 179:1591-7. doi: 10.1002/ajmg.a.61201
Conflict of Interest: The authors declare that the research was conducted in the absence of any commercial or financial relationships that could be construed as a potential conflict of interest.

Copyright (®) 2020 Budny, Zemojtel, Kaluzna, Gut, Niedziela, Obara-Moszynska, Rabska-Pietrzak, Karmelita-Katulska, Stajgis, Ambroziak, Bednarczuk, Wrotkowska, Bukowska-Olech, Jamsheer, Ruchala and Ziemnicka. This is an open-access article distributed under the terms of the Creative Commons Attribution License (CC BY). The use, distribution or reproduction in other forums is permitted. provided the original author(s) and the copyright owner(s) are credited and that the original publication in this journal is cited, in accordance with accepted academic practice. No use, distribution or reproduction is permitted which does not comply with these terms. 